\title{
ELEMENTS OF FINITE ORDER IN FREE PRODUCT SIXTH-GROUPS
}

\author{
by JAMES MCCOOL $\dagger$
}

(Received 17 August, 1967)

Introduction. A free product sixth-group (FPS-group) is, roughly speaking, a free product of groups with a number of additional defining relators, where, if two of these relators have a subword in common, then the length of this subword is less than one sixth of the lengths of either of the two relators.

Britton $[1,2]$ has proved a general algebraic result for FPS-groups and has used this result in a discussion of the word problem for such groups.

In this paper we use the results of [2] to obtain a characterization of the elements of finite order in any FPS-group, and also necessary and sufficient conditions for such a group to be torsion-free. Similar results have been obtained by Greendlinger [3, Theorem VIII] for free sixth-groups, and by Karass, Magnus and Solitar [4] for groups with one defining relation.

The main technical result of this paper (Lemma 5) was suggested by a corresponding result for free sixth-groups proved by Lipschutz [5, Lemma 4].

1. Notation. Statement of the main results.

1.1 Let $\Pi$ be the free product of the set of groups $\left\{G_{\gamma}: \gamma \in \Gamma\right\}$. No restriction is placed on the constituent groups $G_{\gamma}$ of $\Pi$ and the index set $\Gamma$ may be infinite. The non-identity elements of the groups $G_{\gamma}$ are called components of $\Pi$ and are denoted by small letters. We write $x \sim y$ or $x \sim$ ' $y$ according to whether $x$ and $y$ belong to the same constituent group or not. The identity element of $\Pi$ is denoted by $I$ and general elements of $\Pi$ are denoted by capital letters. We write $X . Y$ for the product of the elements $X$ and $Y$ of $\Pi$. Every element $X$ of $\Pi$ except $I$ has a unique normal form expression

$$
X=x_{1} \cdot x_{2} \cdot \ldots \cdot x_{n},
$$

where $n \geqq 1, I \neq x_{i} \in G_{\gamma(i)}(i=1,2, \ldots, n)$ and $\gamma(i) \neq \gamma(i+1)(i=1,2, \ldots, n-1)$. The elements $x_{1}, x_{2}, \ldots, x_{n}$ are called the components of $X$. We write $l(X)=n, \operatorname{In}(X)=x_{1}$ and $\operatorname{Fin}(X)=x_{n}$. We call $l(X)$ the length of $X$ and define the length $l(I)$ of $I$ to be zero.

as

If $X=X_{1}, X_{2} \ldots X_{r}$ and $l(X)=\sum_{i=1}^{r} l\left(X_{i}\right)$, we write $X=X_{1} X_{2} \ldots X_{r} ;$ thus we write(1.11)

$$
X=x_{1} x_{2} \ldots x_{n} \text {. }
$$

Let $X$ have form (1.12). A double segment of $X$ is defined to be any element with form

$$
x_{j}^{\prime} x_{j+1} \ldots x_{s-1} x_{s}^{\prime},
$$

where $1 \leqq j \leqq s \leqq n, x_{j}^{\prime} \sim x_{j}$ and $x_{s}^{\prime} \sim x_{s}$.

$\dagger$ The contents of this paper formed part of a Ph.D. thesis presented to Glasgow University (1966). I should like to thank my supervisor Dr. J. L. Britton for his advice and encouragement, and the Science Research Council for financial support. 
A subword of $X$ is any element with form (1.13), where $x_{j}^{\prime}=x_{j}$ and $x_{s}^{\prime}=x_{s}$. The subword $x_{j} x_{j+1} \ldots x_{s-1} x_{s}$ of $X$ is said to cover the double segment $x_{j}^{\prime} x_{j+1} \ldots x_{s-1} x_{s}^{\prime}$ of $X$. We note that, if $Y$ is a double segment of $X$, then $Y \neq I$. If $l(X) \geqq 2$ and $\operatorname{In}(X) \sim$ ' Fin $(X)$, we define a cyclic arrangement of $X$ to be any element with form $x_{r} x_{r+1} \ldots x_{n} x_{1} x_{2} \ldots x_{r-1}(1 \leqq r \leqq n)$.

We define the number $\beta(X, Y)$ of cancellations and the number $\varepsilon(X, Y)$ of amalgamations in the product $X . Y$ as follows:

Suppose first that $X \neq I$ and $Y \neq I$ and let $X=x_{1} x_{2} \ldots x_{m}, Y=y_{1} y_{2} \ldots y_{n}$. Then

$$
\beta(X, Y)=\left\{\begin{array}{lll}
0 & \text { if } & x_{m}, y_{1} \neq I, \\
\beta & \text { if } & x_{m}, y_{1}=I,
\end{array}\right.
$$

where $\beta$ is the largest integer for which $x_{m-\beta+1} \ldots x_{m}, y_{1} \ldots y_{\beta}=I$, and

$$
\varepsilon(X, Y)= \begin{cases}1 & \text { if } \beta<\operatorname{Min}(m, n) \text { and } x_{m-\beta} \sim y_{\beta+1}, \\ 0 & \text { otherwise. }\end{cases}
$$

If either $X=I$ or $Y=I$, we define $\beta(X, Y)$ and $\varepsilon(X, Y)$ to be zero. Note that

$$
l(X . Y)=l(X)+l(Y)-2 \beta(X, Y)-\varepsilon(X, Y) \text {. }
$$

We put

$$
\alpha(X, Y)=\beta(X, Y)+\varepsilon(X, Y) .
$$

If $X=A . B . C$ and $\alpha\{(A . B), C\}=0$ we write $X=A . B C$; similarly if $\alpha\{A,(B . C)\}=0$ we write $X=A B . C$.

1.2. Let $\Omega$ be a subset of $\Pi$ such that, for all elements $R, R^{\prime}$ of $\Omega$, the following conditions are satisfied:

1) $l(R) \geqq 7$.

(2) $\operatorname{In}(R) \sim^{\prime} \operatorname{Fin}(R)$.

(3) Every cyclic arrangement of $R$ and $R^{-1}$ belongs to $\Omega$.

(4) Either $R^{\prime}=R^{-1}$ or

$$
6 \alpha\left(R^{\prime}, R\right)<\operatorname{Min}\left(l\left(R^{\prime}\right), l(R)\right) .
$$

(5) $\operatorname{Max} \alpha\left(R_{1}, V\right) \neq 0$, where $R_{1}$ is a cyclic arrangement of $R$ or $R^{-1}$ and $V$ is an element of $\Omega$ such that $R_{1} . V \neq I$.

It is easy to show that, if $\Omega$ satisfies conditions (2) to (5), then condition (1) is also satisfied. [Let $R \in \Omega$. By (5) there exist $R_{1}$ (a cyclic arrangement of $R$ or $R^{-1}$ ) and $V \in \Omega$ such that $R_{1} . V \neq I$ and $\alpha\left(R_{1}, V\right) \neq 0$. By (4) we have $6 \leqq 6 \alpha\left(R_{1}, V\right)<l\left(R_{1}\right)=l(R)$.] Thus the conditions we have imposed on the set $\Omega$ are the same as those of [2].

Let [ $\Omega$ ] be the normal subgroup of $\Pi$ generated by the set $\Omega$ and let $\phi$ be the natural homomorphism of $\Pi$ onto $\Pi /[\Omega]$. We denote by $|X|$ the image under $\phi$ of the element $X$ of $\Pi$. If $X$ and $Y$ are elements of $\Pi$ such that $|X|=|Y|$, then we write $X \approx Y$; it is clear that $\approx$ is a congruence relation on $\Pi$. We note that, if $W$ is an element of $\Pi$ such that $W \in[\Omega]$, then $|W|=|I|$ and so $W \approx I$. 
DEFINITION, An FPS-group is defined to be any group isomorphic to a quotient group $\Pi /[\Omega]$ of a free product $\Pi$ of groups, where $\Omega$ is a subset of $\Pi$ such that the five conditions stated above are satisfied.

We now state the main theorem of [2].

THEOREM 1. Let $\Pi /[\Omega]$ be an FPS-group. If $W$ is an element of $\Pi$ such that $W \approx I$ but $W \neq I$, then

(i) there exist elements $X, Y, Z, T$ of $\Pi$ such that $W=X Y Z, Y T^{-1} \in \Omega$ and

$$
l(X . T . Z)<l(W),
$$

(ii) $l(W) \geqq l_{0}=\operatorname{Min}\{l(R): R \in \Omega\}$, and $l(W)=l_{0}$ implies $W \in \Omega$.

From (ii) it follows that the intersection of [ $\Omega$ ] with any constituent group consists only of the identity element. Thus, if $U$ is an element of $\Pi$ of length one, we have $U \approx I$, and if $U^{\lambda} \approx I$ for any integer $\lambda$, then $U^{\lambda}=I$.

Definition. An element $V$ of a group $G$ is said to be a proper power if $V$ is not the identity and there exist an element $J$ of $G$ and an integer $\lambda$ greater than one such that $V=J^{\lambda}$.

We shall prove the following theorems.

THEOREM 2. Let $\Pi /[\Omega]$ be an FPS-group. If $\Omega$ contains a proper power $R, R=J^{\lambda}(\lambda>1)$, then $|J|$ has order $\lambda$ in $\Pi /[\Omega]$.

THEOREM 3. Let $\Pi /[\Omega]$ be an FPS-group. Then $\Pi /[\Omega]$ is torsion-free if and only if

(i) each constituent group of $\Pi$ is torsion-free, and

(ii) no element of $\Omega$ is a proper power.

Arising from these results we have the following characterization of the elements of finite order in any FPS-group.

COROLlaRY 1. Let $\Pi /[\Omega]$ be an FPS-group. If $A$ is an element of $\Pi$ such that $A \approx I$ and $|A|$ has finite order in $\Pi /[\Omega]$, then either

(i) $|A|$ is the image under $\phi$ of a conjugate of an element of finite order in a constituent group of $\Pi$, and has the same order as this element, or

(ii) there exist $J \in \Pi, R \in \Omega$ and positive integers $m, n$ such that $R=J^{n},|A|$ is conjugate to $\left|J^{m}\right|$, and $|A|$ has order $n / t$, where $t$ is the highest common factor of $m$ and $n$.

These results may be summarised as stating that the only elements of finite order in an FPS-group are the obvious ones.

2. Preliminary definitions and results.

2.1. Let $X_{1}, X_{2}, \ldots, X_{r}$ be a sequence of elements of $\Pi$. The sequence of components of $\Pi$ obtained by writing down successively the components of the $X_{i}$ is called the component sequence of $X_{1}, X_{2}, \ldots, X_{r}$. Thus, if $X_{1}=a b c$ and $X_{2}=c^{-1} b$, the component sequence of $X_{1}, X_{2}$ is $a, b, c, c^{-1}, b$. 
ELEMENTS OF FINITE ORDER IN FREE PRODUCT SIXTH-GROUPS 131

Definition. $\dagger$ A partition is a sequence $X_{1}, X_{2}, \ldots, X_{r}(r \geqq 1)$ of elements of $\Pi$ such that the following two conditions are satisfied.

(a) At least one $X_{i}$ is different from $I$.

(b) If the component sequence of $X_{1}, X_{2}, \ldots, X_{r}$ is bracketed so that the terms inside a bracket belong to the same constituent group and terms in adjacent brackets belong to different constituent groups, then the product of the terms in each bracket is not the identity.

For example, if $X_{1}=a b c, X_{2}=c^{-1}, X_{3}=c d, X_{4}=I$, then the bracketing of the component sequence of $X_{1}, X_{2}, X_{3}, X_{4}$ is $\{a\},\{b\},\left\{c, c^{-1}, c\right\},\{d\}$ and so $X_{1}, X_{2}, X_{3}, X_{4}$ is a partition. Here $X_{1}, X_{2}$ is not a partition, since the bracketing is $\{a\},\{b\},\left\{c, c^{-1}\right\}$.

If $X_{1}, X_{2}, \ldots, X_{r}$ is a partition and $X_{1} . X_{2} \ldots . X_{r}=X$, we say that $X_{1}, X_{2}, \ldots, X_{r}$ is a partition of $X$, or just that $X_{1}, X_{2} \ldots \ldots X_{r}$ is a partition of $X$. We note that in this case $X \neq I$, for the length of $X$ is clearly the number of brackets occurring in the bracketing described in (b) above, and this is non-zero since at least one of the $X_{i}$ has non-zero length.

Remark. In Lemma 1 we give two general ways in which we can alter a partition of $X$ to obtain a different partition of $X$. These arise from the following ways in which we can alter the component sequence $d_{1}, d_{2}, \ldots, d_{n}$ of a partition of $X$ so that the component sequence obtained still satisfies condition (b) above. Thus we can

(i) replace two adjacent terms $d_{p}, d_{p+1}$ in the same bracket by their product, provided it is not the identity,

(ii) delete the pair $d_{p}, d_{p+1}$ if their product is the identity,

(iii) replace the term $c$ by the pair of terms $c_{1}, c_{2}$ if $c=c_{1}, c_{2}$ (Note that the notation implies that $c_{1}$ and $c_{2}$ are non-identity elements of the same constituent group as $c$.),

(iv) insert a pair $a, a^{-1}$ before or after the term $d$, provided that $a \sim d$.

We note that, if $X \neq I, X=a_{1} a_{2} \ldots a_{n}$, say, then the sequence consisting of $X$ alone is a partition of $X$ with component sequence $a_{1}, a_{2}, \ldots, a_{n}$. It is clear that the component sequence of any partition of $X$ can be reduced to that of $X$ by a finite number of changes of types (i) and (ii) in the remark above. Conversely, the component sequence of any partition of $X$ can be obtained from that of $X$ by a finite number of changes of types (iii) and (iv) above.

Lemma 1. Let $X_{1}, X_{2}, \ldots, X_{r}$ be a partition of $X$. We have the following results.

(i) If $X_{i}, X_{i+1}=Y$, then $X_{1}, \ldots, X_{i-1}, Y, X_{i+2}, \ldots, X_{r}$ is a partition of $X$.

(ii) If $Y_{1}, Y_{2}, \ldots, Y_{n}$ is a partition of $X_{i}$, then $X_{1}, \ldots, X_{i-1}, Y_{1}, \ldots, Y_{n}, X_{i+1}, \ldots, X_{r}$ is a partition of $X$.

(iii) If $X \neq I$, then $X_{i}$ is a double segment of $X$.

† This definition is based on a similar one used by J. L. Britton in some unpublished work. 
Proof. We prove the results under the assumption that none of the $X_{i}$ is the identity. It is easy to see that the results continue to hold when this restriction is removed.

$1^{0}$. To prove (i), we let $X_{i}=a_{1} \ldots a_{m}, X_{i+1}=b_{1} \ldots b_{n}$. We consider separately the cases $a_{m} \sim b_{1} ; a_{m} \sim b_{1}$ but $a_{m} . b_{1} \neq I ; a_{m} . b_{1}=I$.

If $a_{m} \sim{ }^{\prime} b_{1}$ then the component sequence of $X_{1}, \ldots, X_{i-1}, Y, X_{i+2}, \ldots, X_{r}$ is just the component sequence of $X_{1}, \ldots, X_{r}$, so that (i) holds in this case.

If $a_{m} \sim b_{1}$ but $a_{m} . b_{1} \neq I$, then the component sequence of $X_{1}, \ldots, X_{i-1}, Y, X_{i+2}, \ldots, X_{r}$ is obtained from that of $X_{1}, \ldots, X_{r}$ by replacing the pair of terms $a_{m}, b_{1}$ by their product. Hence (i) holds in this case.

Finally, if $a_{m} \cdot b_{1}=1$, then at least one of $X_{i}, X_{i+1}$ has length one (for if they both had length greater than one the bracket $\left\{a_{m}, b_{1}\right\}$ would occur in the bracketing of the component sequence of $X_{1}, \ldots, X_{r}$ and so we could not have $\left.a_{m} . b_{1}=I\right)$. We suppose firstly that not both $l\left(X_{i}\right)=1$ and $l\left(X_{i+1}\right)=1$ hold. We take $l\left(X_{i}\right)=1, l\left(X_{i+1}\right)>1$; the case $l\left(X_{i}\right)>1, l\left(X_{i+1}\right)=1$ is similar. Thus $X_{i}=a_{1}, X_{i+1}=a_{1}^{-1} b_{2} \ldots b_{n}$ and so $Y=b_{2} \ldots b_{n}$. Hence the component sequence of $X_{1}, \ldots, X_{i-1}, Y, X_{i+2}, \ldots, X_{r}$ is obtained from that of $X_{1}, \ldots, X_{r}$ by deleting the pair of terms $a_{1}, a_{1}^{-1}$, so that (i) holds in this case. Now suppose that $l\left(X_{i}\right)=l\left(X_{i+1}\right)=1$, so that $X_{i}=a_{1}, X_{i+1}=a_{1}^{-1}$. Then by a similar argument we see that (i) also holds in this case. This completes the proof of (i).

$2^{0}$. For (ii) we note that, if the terms due to $X_{i}$ in the component sequence of $X_{1}, \ldots, X_{r}$ are replaced by the terms due to $Y_{1}, \ldots, Y_{n}$, then the component sequence obtained satisfies condition (b) in the definition of a partition, since the new sequence can be obtained from the original one by a finite number of changes of types (iii) and (iv) in the remark above. This proves (ii).

$3^{0}$. We shall indicate the proof of (iii) for the case when $r \geqq 3$ and $i \neq 1, r$. The proofs of the other cases are similar.

From (i) of the lemma it follows that, if $Y=X_{1} \ldots \ldots X_{i-1}$ and $Z=X_{i+1} \ldots \ldots X_{r}$, then $Y . X_{i} . Z$ is a partition of $X$. Let $Y, X_{i}, Z$ have normal forms

$$
Y=a_{1} \ldots a_{l}, \quad X_{i}=b_{1} \ldots b_{m}, \quad Z=c_{1} \ldots c_{n} .
$$

If $a_{l} \sim{ }^{\prime} b_{1}$ and $b_{m} \sim^{\prime} c_{1}$, then $X_{i}$ is a subword of $X$, so that (iii) holds in this case. If $a_{l} \sim b_{1}$ and $b_{m} \sim{ }^{\prime} c_{1}$, then the normal form of $X$ is

$$
a_{1} \ldots a_{l-1}\left(a_{l}, b_{1}\right) b_{2} \ldots b_{m} c_{1} \ldots c_{n},
$$

for $a_{1}, b_{1} \neq I$ since $Y, X_{i}, Z$ is a partition. Clearly $X_{i}$ is a double segment of $X$ in this case. A similar argument shows that (iii) holds if $a_{l} \sim^{\prime} b_{1}$ and $b_{m} \sim c_{1}$. Thus to prove (iii) we need only consider the case when $a_{l} \sim b_{1}$ and $b_{m} \sim c_{1}$. Then, if $m \neq 1$, the normal form of $X$ is

$$
a_{1} \ldots a_{l-1}\left(a_{l} . b_{1}\right) b_{2} \ldots b_{m-1}\left(b_{m} \cdot c_{1}\right) c_{2} \ldots c_{n},
$$

where $a_{l}, b_{1} \neq I$ and $b_{m} . c_{1} \neq I$, since $Y, X_{i}, Z$ is a partition, while, if $m=1$, then $X$ has normal form

$$
a_{1} \ldots a_{l-1}\left(a_{l}, b_{1}, c_{1}\right) c_{2} \ldots c_{n}
$$

and $a_{l} \cdot b_{1} \cdot c_{1} \neq I$, since $Y, X_{i}, Z$ is a partition. In both these cases it is easily seen that $X_{i}$ is a double segment of $X$. This completes the proof of (iii). 
Definition. Let $Y_{1}, \ldots, Y_{i-1}, Y_{i}, Y_{i+1}, \ldots, Y_{n}$ be a partition of $R \in \Omega$. The element $\left(Y_{1} \ldots \ldots Y_{i-1}\right)^{-1}\left(Y_{i+1} \ldots \ldots Y_{n}\right)^{-1}$ of $\Pi$ is called the complement of $Y_{i}$ with respect to the partition $Y_{1}, \ldots, Y_{n}$ of $R$. We write

$$
\left(Y_{1}, \ldots Y_{i-1}\right)^{-1}\left(Y_{i+1} \ldots, Y_{n}\right)^{-1}=\mathscr{C}\left(Y_{i} ; Y_{1}, \ldots, Y_{n}\right) .
$$

Note that $\alpha\left\{\left(Y_{1} \ldots \ldots Y_{i-1}\right)^{-1},\left(Y_{i+1} \ldots Y_{n}\right)^{-1}\right\}=0$, since $\operatorname{In}(R) \sim^{\prime}$ Fin $(R)$ and

$$
\left(Y_{1} \ldots . Y_{i-1}\right), Y_{i},\left(Y_{i+1} \ldots . Y_{n}\right)
$$

is a partition of $R$. If no ambiguity arises concerning the partition being referred to, we may speak of $\left(Y_{1} \ldots Y_{i-1}\right)^{-1}\left(Y_{i+1} \ldots Y_{n}\right)^{-1}$ as the complement of $Y_{i}$ with respect to $R$ and denote it by $\mathscr{C}\left(Y_{i} ; R\right)$. We note that $\mathscr{C}\left(Y_{i} ; R\right) \approx Y_{i}$, since $R \approx I$.

Lemma 2. Let $A, Y, B$ be a partition of $R \in \Omega$ and $C, Y, D$ a partition of $R^{\prime} \in \Omega$. Suppose that one of the following three conditions is satisfied.

(i) $R=A . Y B, R^{\prime}=C . Y D$ and $l(Y) \geqq \frac{1}{6} \operatorname{Min}\left(l(R), l\left(R^{\prime}\right)\right)$.

(ii) $R=A Y . B, R^{\prime}=C Y . D$ and $l(Y) \geqq \frac{1}{6} \operatorname{Min}\left(l(R), l\left(R^{\prime}\right)\right)$.

(iii) $l(Y) \geqq \frac{1}{6} \operatorname{Min}\left(l(R), l\left(R^{\prime}\right)\right)+1$.

Then $\mathscr{C}(Y ; A, Y, B)=\mathscr{C}(Y ; C, Y, D)$.

Proof. $1^{\circ}$. Suppose that (i) holds. Then $\beta(A, Y)=0$, since $A, Y, B$ is a partition and $\alpha\{(A . Y), B\}=0$. Similarly $\beta(C, Y)=0$. Let $R_{1}=B A . Y, R_{1}^{\prime}=D C . Y$. Then $R_{1}$ and $R_{1}^{\prime}$ belong to $\Omega$ and

$$
\alpha\left(R_{1}^{\prime}, R_{1}^{-1}\right) \geqq l(Y) \geqq \frac{1}{6} \operatorname{Min}\left(l\left(R^{\prime}\right), l\left(R_{1}^{-1}\right)\right) .
$$

Hence $R_{1}^{\prime}=R_{1}$ and so $B A=D C$, which proves the lemma in this case. A similar argument proves the lemma if condition (ii) holds.

$2^{0}$. Suppose that condition (iii) holds. Then $Y \neq I$ and $Y$ is a double segment of $R$ and $R^{\prime}$, since $A, Y, B$ and $C, Y, D$ are partitions. Let $Y=Y_{1} Y_{2}$, where $Y_{2}=$ Fin $(Y)$. Then $R=A . Y_{1}\left(Y_{2} \cdot B\right), R^{\prime}=C \cdot Y_{1}\left(Y_{2} \cdot D\right), l\left(Y_{1}\right) \geqq \frac{1}{6} \operatorname{Min}\left(l(R), l\left(R^{\prime}\right)\right)$ and so (i) of the lemma is satisfied with $Y_{1}$ in place of $Y$ and $Y_{2} . B, Y_{2} . D$ in place of $B$ and $D$ respectively. Hence $Y_{2} . B A=Y_{2} . D C$ and so $B A=D C$. This completes the proof of the lemma.

2.2. Let $S$ be a subword of $R \in \Omega$ such that $l(S) \geqq\left[\frac{1}{2} l(R)\right]$ (where the square brackets denote " the integral part of "). We define the subset $\Omega_{1}$ of $\Pi$ to be the set of all such $S$, where $R$ ranges over all the elements of $\Omega$.

We note that, if $S \in \Omega_{1}$, where $S$ is a subword of $R \in \Omega$ say, then

and

$$
l(S) \geqq \frac{2}{6} l(R)
$$

$$
l(S) \geqq \frac{1}{6} l(R)+1 \text {. }
$$

For we can write $l(R)=6 \mu+\lambda$, where $\mu \geqq 1$ and $0 \leqq \lambda \leqq 5$. Then, since $l(S) \geqq\left[\frac{1}{2} l(R)\right]$, we have

$$
l(S) \geqq 3 \mu+\left[\frac{1}{2} \lambda\right] \geqq 2 \mu+1+\left[\frac{1}{2} \lambda\right] \geqq 2 \mu+\frac{1}{3} \lambda=\frac{2}{6} l(R),
$$

so that (2.21) holds. Now (2.22) follows from (2.21) since $l(R) \geqq 7$. 
From property (3) of $\Omega$ it follows that, if $S \in \Omega_{1}$, we can find $R \in \Omega, T \in \Pi$ such that $R=S T$; moreover $R$ and $T$ are unique. For if $S \in \Omega_{1}$, there is an element $R$ of $\Omega$ such that $R=A S B$ for some $A$ and $B$, and $l(S) \geqq\left[\frac{1}{2} l(R)\right]$. Thus $R_{1}=S B A \in \Omega$, and, if $R_{2}=S T \in \Omega$, then

$$
\alpha\left(R_{1}^{-1}, R_{2}\right) \geqq l(S) \geqq \frac{2}{6} l(R)=\frac{2}{6} l\left(R_{1}^{-1}\right),
$$

by (2.21), and it follows that $R_{1}=R_{2}$. We note also that, if $S \in \Omega_{1}$, then we can speak of the complement of $S$, for by (2.21) and Lemma 2 any two complements of $S$ are equal.

Let $X, Y, Z$ be a partition of $W \in \Pi$ and $A, Y, B$ a partition of $R \in \Omega$, where $Y \neq I$. Put $W^{\prime}=X .\left(A^{-1} B^{-1}\right) . Z$. We say that $Y$ is $\Omega$-replaceable in $W$ and that $W^{\prime}$ is the result of replacing $Y$ by $\mathscr{C}(Y ; A, Y, B)$ in the partition $X . Y . Z$ of $W$. If no ambiguity arises concerning the partitions being referred to, we abbreviate this by saying that $W^{\prime}$ is the result of replacing $Y$ in $W$ by $\mathscr{C}(Y ; R)$. We note that, if $W^{\prime}$ is the result of replacing $Y$ in $W$ by $\mathscr{C}(Y ; R)$, then $W^{\prime} \approx W$, since $Y \approx \mathscr{C}(Y ; R)$.

Two replacements in $W$ are said to be equivalent if they yield the same result. Thus if L.M.N is a partition of $Y$ above, so that $X . L . M . N . Z$ is a partition of $W$ and $A . L . M . N . B$ is a partition of $R$, then replacing $Y$ in $X . Y . Z$ by $A^{-1} B^{-1}$ is equivalent to replacing $M$ in $X . L . M . N . Z$ by $(A . L)^{-1}(N . B)^{-1}$.

Let $Y$ be $\Omega$-replaceable in $W$ with result $W^{\prime}$. If $Y$ is a subword of $W, Y \in \Omega_{1}$ and

$$
l\left(W^{\prime}\right)<l(W),
$$

then we say that $W$ is $\Omega$-reducible by $Y$. If $W \neq I$ and $W$ contains no subword $Y$ by which it is $\Omega$-reducible, then we say that $W$ is $\Omega$-reduced. We note that, if $W$ is $\Omega$-reduced, then so are $W^{-1}$ and any subword of $W$ or $W^{-1}$; for if $W=X . Y . Z$ and $Y$ is $\Omega$-replaceable in $W$ with result $W_{1}$, then $W^{-1}=Z^{-1} \cdot Y^{-1} \cdot X^{-1}$ and $Y^{-1}$ is $\Omega$-replaceable in $W^{-1}$ with result $W_{1}^{-1}$, so that, if $W$ is $\Omega$-reducible by $Y$, then $W^{-1}$ is $\Omega$-reducible by $Y^{-1}$, which proves the first part of the statement. If $W=A B C$ and $B$ is $\Omega$-reducible by some subword $Y, B=X Y Z$ say, then $W=A X Y Z C$ and clearly $W$ is $\Omega$-reducible by $Y$, which proves the second part of the statement.

The following lemma is a restatement of (i) of Theorem 1 together with some consequences of it.

Lemma 3. Let $W$ be any non-identity element of $\Pi$. We have the following results:

(i) If $W \approx I$, then $W$ contains a subword $Y$ by which it is $\Omega$-reducible.

(ii) If $W$ is $\Omega$-reduced, then $W \approx I$.

(iii) If $W \approx I$, then there exists an element $W^{\prime}$ of $\Pi$ such that $W^{\prime}$ is $\Omega$-reduced, $W^{\prime} \approx W$ and $l\left(W^{\prime}\right) \leqq l(W)$.

Proof. (i) is a restatement of (i) of Theorem 1, and (ii) follows immediately from (i) and the definition of an $\Omega$-reduced element.

To prove (iii) we note that $W^{\prime}=W$ will do if $W$ is $\Omega$-reduced. If $W$ is not $\Omega$-reduced, then $W$ contains a subword $Y$ by which it is $\Omega$-reducible. Let $W_{1}$ be the result of replacing $Y$ in $W$. Then $W_{1} \approx W$ and $l\left(W_{1}\right)<l(W)$. If $W_{1}$ is $\Omega$-reduced, then we can take $W^{\prime}=W_{1}$; 


\section{ELEMENTS OF FINITE ORDER IN FREE PRODUCT SIXTH-GROUPS 135}

if not, then we carry out the above process with $W_{1}$ in place of $W$ to obtain an element $W_{2}$ such that $W_{2} \approx W_{1}$ and $l\left(W_{2}\right)<l\left(W_{1}\right)$. Repeating the process if necessary, we must eventually obtain an element $W^{\prime}$ satisfying (iii) of the lemma, since $I \approx W \approx W_{1} \approx W_{2} \approx \ldots$ and $l(W)>l\left(W_{1}\right)>l\left(W_{2}\right)>\ldots$

Let $W$ be $\Omega$-reducible by $Y$, where $W=X Y Z, Y T^{-1}=R \in \Omega$ say. Let $W^{\prime}$ be the result of replacing $Y$ in $W$, so that $W^{\prime}=X . T$. $Z$. Then, if $\beta(X, T)=\beta(T, Z)=0$, we say that $Y$ is maximal in $W$. We note that, if $W$ is $\Omega$-reducible by $Y$, then we can find a subword $S$ of $W$ such that $W$ is $\Omega$-reducible by $S, Y$ is a subword $S, S$ is maximal in $W$ and replacing $S$ in $W$ is equivalent to replacing $Y$ in $W$. \{One way of choosing such an $S$ is as follows. We have

$$
W=X Y Z, \quad Y \in \Omega_{1}, \quad Y T^{-1} \in \Omega, \quad W^{\prime}=X . T . Z .
$$

We can find elements $X_{1}, X_{2}$ of $\Pi$ such that

$$
W=X_{1} X_{2} Y Z \text { and } \beta(X, T)=l\left(X_{2}\right) .
$$

Then $T=X_{2}^{-1} T_{1}$ for some $T_{1} \in \Pi, X_{2} Y T_{1}^{-1} \in \Omega$, since $Y T_{1}^{-1} X_{2} \in \Omega, X_{2} Y \in \Omega_{1}, W$ is $\Omega$ reducible by $X_{2} Y$ and $\beta\left(X_{1}, T_{1}\right)=0$. The result of replacing $X_{2} Y$ in $W$ is $X_{1}, T_{1}, Z$. We can find $Z_{1}, Z_{2}$ such that

$$
W=X_{1} X_{2} Y Z_{1} Z_{2} \quad \text { and } \quad \beta\left(T_{1}, Z\right)=l\left(Z_{1}\right) .
$$

Then $T_{1}=T_{2} Z_{1}^{-1}$ for some $T_{2} \in \Pi, X_{2} Y Z_{1} T_{2}^{-1} \in \Omega, X_{2} Y Z_{1} \in \Omega_{1}, W$ is $\Omega$-reducible by $X_{2} Y Z_{1}$ and $\beta\left(X_{1}, T_{2}\right)=\beta\left(T_{2}, Z_{2}\right)=0\left(\beta\left(X_{1}, T_{2}\right)=0\right.$ since $\beta\left(X_{1}, T_{1}\right)=0$ and either $T_{2}=I$ or $\left.\operatorname{In}\left(T_{1}\right)=\operatorname{In}\left(T_{2}\right)\right)$. Clearly $X_{2} Y Z_{1}$ has the properties required of $S$. $\}$

In view of this, if $W$ is $\Omega$-reducible by $Y$, we shall usually choose the $\Omega$-replaceable subword $Y$ maximally. We note further that, if $Y$ is maximal in $W$, then, with the above notation, $X . T . Z$ will be a partition unless either

$$
T=\dot{I} \text { and } \beta(X, Z)>0
$$

or

$$
l(T)=1, \quad \varepsilon(X, T)=\varepsilon(T, Z)=1 \text { and } \operatorname{Fin}(X) \cdot T \cdot \operatorname{In}(Z)=I .
$$

We now introduce the concepts of cyclic length and cyclic $\Omega$-reduction. Let $W$ be any element of $\Pi$. We define the cyclic length of $W$, written $l^{\circ}(W)$, by

$$
l^{0}(W)=\operatorname{Min}\{l(X): X \text { is a conjugate of } W \text { in } \Pi\} .
$$

It follows easily that any two conjugate elements of $\Pi$ have the same cyclic length and that, if $l(W) \geqq 2$ and $\operatorname{In}(W) \sim^{\prime}$ Fin $(W)$, then $l^{\circ}(W)=l(W)$.

Definition. An element $W$ of $\Pi$ is said to be cyclically $\Omega$-reduced if either $l(W)=1$, or $l(W)>1$ and $W$ satisfies the following conditions:

(i) $\operatorname{In}(W) \sim$ ' Fin $(W)$.

(ii) If $Y \in \Omega_{1}$ is a subword of $W$ and $W^{\prime}$ is the result of replacing $Y$ in $W$, then

$$
l^{0}\left(W^{\prime}\right) \geqq l(W) \text {. }
$$

(iii) Every cyclic arrangement of $W$ satisfies (ii). 
We note that if $W$ is cyclically $\Omega$-reduced, then $W$ is $\Omega$-reduced and every cyclic arrangement of $W$ and $W^{-1}$ is cyclically $\Omega$-reduced.

\section{The main result.}

3.1. Before proving the main technical result of this paper we need a lemma concerning commuting elements of $\Pi$.

LEMMA 4. Let $A, B$ be elements of $\Pi$ such that $\alpha(A, B)=\alpha(B, A)=0$. If $A B=B A$, then there exist $J \in \Pi$ and integers $\lambda, \mu$ such that $A=J^{\lambda}, B=J^{\mu}$ and $\alpha(J, J)=0$.

Proof. Put $m=l(A B)$. We prove the lemma by induction on $m$. The result holds for $m=0$, since then $A=B=I$ and we can take $J=I, \lambda=\mu=1$. Assume that $m>0$ and that the result holds for all pairs $A_{1}, B_{1}$ satisfying the requirements of the lemma and for which $l\left(A_{1} B_{1}\right)<m$. We suppose also, without loss of generality, that $l(A) \leqq l(B)$. Then, since $A B=B A$, we have, from the uniqueness of the normal form of the elements of a free product, that there exists an element $C$ of $\Pi$ such that $B=A C$. Hence $A A C=A C A$ and so $A C=C A$. If $A \neq I$, then $l(A C)<m$ and so by the induction hypothesis there exist $J \in \Pi$ and integers $\lambda, \mu$ such that $A=J^{\lambda}, C=J^{\mu}$ and $\alpha(J, J)=0$. Thus $B=J^{\lambda+\mu}$ and the result holds for the pair $A, B$ in this case. If $A=I$, then we can take $J=B, \lambda=0$ and $\mu=1$. Hence the result holds for the pair $A, B$. This proves the lemma.

Now we come to the main result.

LEMMA 5. Let $W$ be cyclically $\Omega$-reduced and $l(W)>1$.

(A). If $W^{2}$ is cyclically $\Omega$-reduced, then either

(i) $W^{n}$ is $\Omega$-reduced for all integers $n$, or

(ii) there exist $J \in \Pi, R \in \Omega$ and non-zero integers $\sigma, \tau$ such that $W=J^{\sigma}, R=J^{t}$.

(B). If $W^{2}$ is not cyclically $\Omega$-reduced, then either

(iii) there exist $R \in \Omega, T \in \Pi$ and a cyclic arrangement $W_{1} W_{2}$ of $W$ such that

$$
R=W_{1} W_{2} W_{1} T^{-1},
$$

$\left(T . W_{2}\right)^{n}$ is $\Omega$-reduced for all integers $n$ and $\left(T . W_{2}\right),\left(T . W_{2}\right)^{2}, \ldots$ are increasing in length, or

(iv) part (ii) of (A) above holds.

Note. We note that, if (iii) holds, then $T . W_{2}$ is the result of replacing $W_{1} W_{2} W_{1}$ by $T$ in $\left(W_{1} W_{2} W_{1}\right) W_{2}$, so that $T . W_{2} \approx\left(W_{1} W_{2}\right)^{2}$. Now $\left(W_{1} W_{2}\right)^{2}$ is conjugate to $W^{2}$, since $W_{1} W_{2}$ is a cyclic arrangement of $W$, and so $\left|T . W_{2}\right|$ is conjugate to $|W|^{2}$ in $\Pi /[\Omega]$.

It follows that, if either (i) or (iii) holds, then $|W|$ has infinite order in $\Pi /[\Omega]$, while, if (ii) holds, then $|W|$ has finite order.

Proof of $(A) .1^{0}$. We have $W^{2}$ cyclically $\Omega$-reduced. If $W^{n}$ is $\Omega$-reduced for all positive integers $n$, then the result follows; so we assume that $W^{m}$ is not $\Omega$-reduced for some positive integer $m$. 


\section{ELEMENTS OF FINITE ORDER IN FREE PRODUCT SIXTH-GROUPS 137}

Thus we have $W^{m}=X S Y$ say, where $S \in \Omega_{1}$ and is chosen to be maximal, $S T^{-1}=R \in \Omega$, $X S Y \approx X . T . Y$,

and

$$
\beta(X, T)=\beta(T, Y)=0
$$

$$
l(X S Y)>l(X . T . Y) \text {. }
$$

$2^{0}$. We show $l(S) \geqq l\left(W^{2}\right)$.

For suppose that this is not the case. Then there exists a cyclic arrangement $W^{\prime}$ of $W$ such that $W^{\prime 2}=S P$ for some $P \neq I$. We note that neither $X=I$ nor $Y=I$ can hold, since otherwise, as $l(S)<l\left(W^{2}\right)$, we could take $m=2$ and then (3.11) and (3.12) would contradict the fact that $W^{2}$ is cyclically $\Omega$-reduced. Thus $\operatorname{Fin}(X)=\operatorname{Fin}(P)=a, \operatorname{In}(Y)=\operatorname{In}(P)=b$, say. From (3.11) and (3.12) we have either

(i) $l(S)>l(T)$, or

(ii) $l(S)=l(T)$ and one of $\varepsilon(a, T), \varepsilon(T, b)$ is non-zero, or

(iii) $l(S)=l(T)-1$ and $\varepsilon(a, T)=\varepsilon(T, b)=1$.

But each of (i), (ii) and (iii) above contradicts the fact that $W^{2}$ is cyclically $\Omega$-reduced, as is easily seen by replacing $S$ by $T$ in $W^{\prime 2}=S P$. Hence $l(S) \geqq l\left(W^{2}\right)$.

$3^{0}$. It follows that there exist a cyclic arrangement $A B$ of $W$ and an integer $r \geqq 2$ such that

$$
S=(A B)^{r} A,
$$

where without loss of generality we may take $B \neq I$. Thus $R=(A B)^{r} A T^{-1}$, and since $S \in \Omega_{1}$ we have

We show that

$$
l\left\{(A B)^{r} A\right\} \geqq\left[\frac{1}{2} l(R)\right] .
$$

$$
l\left\{(A B)^{r-1}\right\} \geqq \frac{1}{6} l(R) .
$$

Suppose that $l\left\{(A B)^{r-1}\right\}<\frac{1}{6} l(R)$. Then $l\left\{(A B)^{r-1}\right\} \leqq\left[\frac{1}{b} l(R)\right]$. Frcm (3.13) we have

$$
\left[\frac{1}{2} l(R)\right] \leqq l\left\{(A B)^{r} A\right\}=l\left\{(A B)^{r-1}\right\}+l(A B)+l(A)<3\left[\frac{1}{6} l(R)\right]
$$

(the last inequality on the right hand side is strict, since $l(A)<l(A B)$ as $B \neq I)$. This is a contradiction, hence (3.14) must hold.

Now $(A B)^{r-1} A T^{-1} A B$ and $(A B)^{r-1} A B A T^{-1}$ are elements of $\Omega$, and from (3.14) and property (4) of $\Omega$ it follows that

and so

$$
(A B)^{r-1} A T^{-1} A B=(A B)^{r-1} A B A T^{-1}
$$

$$
A T^{-1} A B=A B A T^{-1} \text {. }
$$

It now follows from Lemma 4 that there exist $J \in \Pi$ and integers $\lambda$, $\mu$ such that

$$
\alpha(J, J)=0, \quad A B=J^{\lambda}, \quad A T^{-1}=J^{\mu}
$$

and so

$$
R=J^{\lambda+\mu} \text {. }
$$


Since $A B \neq I$ and $R \neq I$, it is clear that $J \neq I$ and that the integers $\lambda$ and $r \lambda+\mu$ are non-zero. Now $W$ is a cyclic arrangement of $A B$ and it is easy to see that there exist elements $J_{1}, J_{2}$ of $\Pi$ such that $J=J_{1} J_{2}$ and $W=\left(J_{2} J_{1}\right)^{\lambda}$. Now $R_{1}=\left(J_{2} J_{1}\right)^{r \lambda+\mu}$ is an element of $\Omega$, and so we see that (ii) of the lemma holds. This concludes the proof of (A).

Proof of $(B) .1^{0}$. We have $l(W)>1, W$ cyclically $\Omega$-reduced and $W^{2}$ not cyclically $\Omega$-reduced. It follows that there exist a cyclic arrangement $U$ of $W$, an element $V$ of $\Pi$ and an element $S$ of $\Omega_{1}$ such that

and

$$
U^{2}=S V, \quad R=S T^{-1} \in \Omega
$$

$$
l^{0}(T . V)<l\left(U^{2}\right) .
$$

It is clear that by choosing $U$ and $S$ suitably we can ensure in addition that

$$
\beta(T, V)=\beta(V, T)=0 .
$$

(Suppose, for example, that $\beta(V, T)=r$, so that $V=V_{1} V_{2}, T=V_{2}^{-1} T_{1}$, where $l\left(V_{2}\right)=r$ and $\beta\left(V_{1}, T_{1}\right)=0$. Then $U^{2}=S V_{1} V_{2}$ and $R=S T_{1}^{-1} V_{2}$. It is easy to see that we can find a cyclic arrangement $U_{1}$ of $W$ such that $U_{1}^{2}=V_{2} S V_{1}$. Now $R_{1}=V_{2} S T_{1}^{-1} \in \Omega, V_{2} S \in \Omega_{1}$,

$$
\left.l^{\circ}\left(T_{1}, V_{1}\right)=l^{0}(T . V)<l\left(U^{2}\right)=l\left(U_{1}^{2}\right) \quad \text { and } \quad \beta\left(V_{1}, T_{1}\right)=0 .\right)
$$

$2^{0}$. We now show that

and

$$
\text { if } V=I \text {, then (ii) of the lemma holds, }
$$

$$
\text { if } l(V)=1 \text {, then } \varepsilon(T, V)=0 .
$$

We suppose that either $V=I$ or $l(V)=1$ and $\varepsilon(T, V)=1$. Then we can write

where $\beta\left(U, T_{1}^{-1}\right)=0$ and

$$
R=U^{2} \cdot T_{1}^{-1} \text {, }
$$

$$
l^{0}\left(T_{1}\right)<l\left(U^{2}\right) .
$$

FFor, if $V=I$, then we can take $T_{1}=T$, while, if $l(V)=1$ and $\varepsilon(T, V)=1$, then

$$
R=S T^{-1}=\left(U^{2} \cdot V^{-1}\right) T^{-1}=U^{2} \cdot\left(V^{-1} \cdot T^{-1}\right)=U^{2} \cdot T_{1}^{-1},
$$

where $T_{1}=T . V$, Fin $(U) \cdot \operatorname{In}\left(T_{1}^{-1}\right)=V \cdot V^{-1} \cdot \operatorname{In}\left(T^{-1}\right)$, so that $\beta\left(U, T_{1}^{-1}\right)=0$, and

$$
\left.l^{0}\left(T_{1}\right)=l^{0}(T . V)<l\left(U^{2}\right) .\right\}
$$

Now, if $T_{1}=I$, then $R=U^{2}$ by (3.19), and, since $U$ is a cyclic arrangement of $W$, this is clearly equivalent to (ii) of the lemma. (Note also that, if $T_{1}=I$, then $\varepsilon(T, V)=0$.) Hence we can assume that $T_{1} \neq I$. Then we can write $T_{1}^{-1}=T_{2}^{-1} A T_{2}$ say, where either $l(A)=1$ or $l(A)>1$ and $\beta(A, A)=0$. We show that

$$
l\left(T_{2}\right)<\frac{1}{6} l(R) .
$$

For suppose that $l\left(T_{2}\right) \geqq \frac{1}{6} l(R)$. Now $R=\left(U^{2} \cdot T_{2}^{-1}\right) A T_{2}$ and so both $A T_{2}\left(U^{2} \cdot T_{2}^{-1}\right)$ and $A^{-1}\left(T_{2} \cdot U^{-2}\right) T_{2}^{-1}$ are elements of $\Omega$. Hence, by property (4) of $\Omega$, we have

so that

$$
A T_{2}\left(U^{2} . T_{2}^{-1}\right)=A^{-1}\left(T_{2} \cdot U^{-2}\right) T_{2}^{-1},
$$

$$
A T_{2} U^{2}=A^{-1} T_{2} \cdot U^{-2}
$$


and in particular $\operatorname{Fin}(U)=\operatorname{Fin}\left(U^{-1}\right)=\{\operatorname{In}(U)\}^{-1}$. This is a contradiction, since $l(U)>1$ and $U$ is cyclically $\Omega$-reduced. Hence (3.21) must hold.

Now from (3.19) we have

$$
l(R)=2 l(U)+l\left(T_{1}\right)-\varepsilon\left(U, T_{1}^{-1}\right)=2 l(U)+l(A)+2 l\left(T_{2}\right)-\varepsilon\left(U, T_{1}^{-1}\right),
$$

so that

and, by (3.21),

$$
2 l(U)+l(A) \geqq l(R)-2 l\left(T_{2}\right),
$$

$$
2 l(U)+l(A)>\frac{4}{6} l(R) .
$$

Also, from (3.20), we have

hence

$$
2 l(U)>l^{0}\left(T_{1}\right)=l^{0}\left(T_{2}^{-1} A T_{2}\right)=l^{0}(A) \geqq l(A)-1 ;
$$

$$
2 l(U) \geqq l(A)
$$

and from (3.22) it follows that

$$
l(U)>\frac{1}{6} l(R) .
$$

Now $U U . T_{1}^{-1}$ and $U . T_{1}^{-1} U$ are elements of $\Omega$ of length $l(R)$; hence

and so

$$
U U . T_{1}^{-1}=U . T_{1}^{-1} U
$$

$$
U . T_{1}^{-1}=T_{1}^{-1} U \text {. }
$$

In particular $l\left(U . T_{1}^{-1}\right)=l\left(T_{1}^{-1} U\right)$, so that $\varepsilon\left(U, T_{1}^{-1}\right)=0$ and therefore

$$
R=U^{2} T_{1}^{-1} \text {. }
$$

Now, if $l(V)=1$ and $\varepsilon(T, V)=1$, then $R=U^{2} \cdot T_{1}^{-1}$ and $\varepsilon\left(U, T_{1}^{-1}\right)=1$, which contradicts (3.24). Hence $T_{1}=T$ and we have proved (3.18). Also, from (3.23) we have $U T^{-1}=T^{-1} U$, and by Lemma 4 there exist $J \in \Pi$ and integers $\lambda$, $\mu$ such that

$$
U=J^{\mu}, \quad T^{-1}=J^{\lambda} \quad \text { and so } \quad R=J^{2 \mu+\lambda} .
$$

Clearly $\mu$ and $2 \mu+\lambda$ are non-zero. Since $U$ is a cyclic arrangement of $W$ the above result is equivalent to (ii) of the lemma, so that we have proved (3.17).

$3^{0}$. In view of (3.17) we can assume for the remainder of the proof that $V \neq I$. Under this assumption we shall show that (iii) of the lemma holds. We note firstly that

For, if $T=I$, then $U^{2}=R V$, so that

$$
T \neq I \text {. }
$$

$$
2 l(U)=l(R)+l(V)>l(R) .
$$

It follows that we can write $U=S_{1} P_{1}$, where $S_{1}$ is a subword of $R$ such that $l\left(S_{1}\right)>\frac{1}{2} l(R)$. Now replacing $S_{1}$ in $U$ by $\mathscr{C}\left(S_{1} ; R\right)$ yields an element whose length is less than $l(U)$, contradicting the fact that $U$ is cyclically $\Omega$-reduced. Hence (3.25) must hold.

We now show that

$$
\text { if } l(T)=1 \text {, then } \varepsilon(T, V)=0 \text {. }
$$


Suppose that $l(T)=1$ and $\varepsilon(T, V)=1$. Then $l(V) \geqq 2$, by (3.18), and

$$
2 l(U)=l\left(U^{2}\right)=l(S)+l(V)=l(R)-1+l(V)>l(R) .
$$

Now $l(S)>\frac{1}{2} l(R)$, since $l(T)=1$, and, since $U^{2}=S V$ and $l(U)>\frac{1}{2} l(R)$, it follows that we can write $U=S_{1} P_{1}$, where $S_{1}$ is a subword of $R$ and $l\left(S_{1}\right)>\frac{1}{2} l(R)$. This yields a contradiction as in the proof of (3.25). Hence (3.26) must hold.

Now $V \neq I$ and $T \neq I$ and in view of (3.16), (3.18) and (3.26) it follows that

$$
l^{0}(T . V)=l(T . V)-\varepsilon(V, T)
$$

for, if $l(V) \geqq 2$ and $l(T) \geqq 2$, then thls is so by (3.16), while, if $l(V)=1$, then it is so by (3.16) and (3.18), and if $l(T)=1$, then it is so by (3.16) and (3.26). Hence instead of (3.15) we can write either

or

$$
l(T . V)<l\left(U^{2}\right)
$$

$$
l(T . V)=l\left(U^{2}\right) \quad \text { and } \quad \operatorname{In}(T . V) \sim \operatorname{Fin}(T . V) .
$$

$4^{0}$. We show that $l(S) \geqq l(U)$.

Suppose that $l(S)<l(U)$. Then $U=S P$ for some $P \neq I$. Hence $V=P S P$ and so $\operatorname{In}(P)=\operatorname{In}(V)$, Fin $(P)=$ Fin $(V)$. From (3.16), (3.27) and (3.28) it follows that either

or

$$
l(T . P)<l(U)
$$

$$
l(T . P)=l(U) \quad \text { and } \quad \operatorname{In}(T . P) \sim \operatorname{Fin}(T . P)
$$

Each of these contradicts the fact that $U$ is cyclically $\Omega$-reduced. Hence we must have $l(S) \geqq l(U)$.

Thus $S$ has form $W_{1} W_{2} W_{1}$, say, where $U=W_{1} W_{2}$, so that $l^{\prime}=W_{2} \neq I$ and

Put $l(R)=\lambda . \quad$ Then

$$
R=W_{1} W_{2} W_{1} T^{-1} \text {. }
$$

since $S \in \Omega_{1}$, and

$$
l\left(W_{1} W_{2} W_{1}\right) \geqq\left[\frac{1}{2} \lambda\right]
$$

$$
l\left(W_{1} W_{2}\right) \leqq \frac{1}{2} \lambda
$$

since $l\left(W_{1} W_{2}\right)>\frac{1}{2} \lambda$ would imply that $U$ was not cyclically $\Omega$-reduced.

Rewriting (3.16), (3.27) and (3.28), we have

and either

$$
\beta\left(T, W_{2}\right)=\beta\left(W_{2}, T\right)=0
$$

or

$$
l\left(T . W_{2}\right)<l\left(U^{2}\right)
$$

$$
l\left(T . W_{2}\right)=l\left(U^{2}\right) \quad \text { and } \quad \operatorname{In}\left(T . W_{2}\right) \sim \operatorname{Fin}\left(T . W_{2}\right) .
$$

$5^{0}$. We now obtain upper bounds for $l\left(W_{1}\right)$ and lower bounds for $l\left(W_{2}\right)$ and $l(T)$.

Suppose that $l\left(W_{1}\right) \geqq \frac{1}{6} \lambda$. Then, using property (4) of $\Omega$, we have

$$
W_{1} W_{2} W_{1} T^{-1}=W_{1} T^{-1} W_{1} W_{2}
$$


and so $\operatorname{In}\left(W_{2}\right)=\operatorname{In}\left(T^{-1}\right)=\{\operatorname{Fin}(T)\}^{-1}$, which contradicts (3.31). Hence

$$
l\left(W_{1}\right)<\frac{1}{6} \lambda .
$$

Moreover, if either In $\left(W_{2}\right) \sim \operatorname{In}\left(T^{-1}\right)$ or Fin $\left(W_{2}\right) \sim$ Fin $\left(T^{-1}\right)$, a similar argument shows that in these cases we must have

We now prove that

$$
l\left(W_{1}\right)<\frac{1}{6} \lambda-1 .
$$

$$
l\left(W_{2}\right)>\frac{1}{6} \lambda .
$$

Suppose firstly that $\varepsilon\left(W_{2}, T\right)=\varepsilon\left(T, W_{2}\right)=0$. Then (3.32) must hold, that is,

$$
l(T)+l\left(W_{2}\right)<l\left(W_{1} W_{2} W_{1} W_{2}\right),
$$

and so $l\left(W_{1} W_{2} W_{1}\right)>\frac{1}{2} \lambda$. From (3.34) it follows that in this case

$$
l\left(W_{2}\right)>\frac{1}{6} \lambda \text {. }
$$

Suppose now that at least one of $\varepsilon\left(W_{2}, T\right), \varepsilon\left(T, W_{2}\right)$ is non-zero. Let $\lambda=6 \sigma+\tau$, where $\sigma \geqq 1$ and $0 \leqq \tau \leqq 5$. From (3.29) we have $l\left(W_{1} W_{2} W_{1}\right) \geqq 3 \sigma+\left[\frac{1}{2} \tau\right]$ and from (3.35) we have $l\left(W_{1}\right)<\sigma+\frac{1}{6} \tau-1$. Hence

$$
\begin{aligned}
l\left(W_{2}\right)=l\left(W_{1} W_{2} W_{1}\right)-2 l\left(W_{1}\right) & >3 \sigma+\left[\frac{1}{2} \tau\right]-2\left(\sigma+\frac{1}{6} \tau-1\right) \\
& =\sigma+1+\frac{1}{6} \tau+\left(1+\left[\frac{1}{2} \tau\right]-\frac{1}{3} \tau\right) \\
& \geqq \sigma+1+\frac{1}{6} \tau=\frac{1}{6} \lambda+1 .
\end{aligned}
$$

Combining (3.37) and (3.38) we see that (3.36) holds.

Now we look at $l(T)$. We have

$$
l(T)=\lambda-l\left(W_{1} W_{2} W_{1}\right)=\lambda-l\left(W_{1} W_{2}\right)-l\left(W_{1}\right)>\lambda-\frac{1}{2} \lambda-\frac{1}{6} \lambda=\frac{1}{3} \lambda,
$$

and since $\lambda \geqq 7$ we have

$$
l(T)>\frac{1}{6} \lambda+1 .
$$

We note that from (3.36) and (3.39) we have $l\left(W_{2}\right) \geqq 2$ and $l(T) \geqq 2$. From (3.31) it follows that $\left(T . W_{2}\right),\left(T . W_{2}\right)^{2}, \ldots$ are increasing in length, and that, for any positive integer $n$, $\left(T . W_{2}\right)^{n}$ is a partition.

$6^{0}$. We complete the proof by showing that $\left(T, W_{2}\right)^{n}$ is $\Omega$-reduced for all positive integers $n$. We suppose that, for some positive integer $n,\left(T . W_{2}\right)^{n}$ contains a subword $Q$ by which it is $\Omega$-reducible, $Q Y^{-1}=R_{1} \in \Omega$ say. We show that either

replacing $Q$ in $\left(T . W_{2}\right)^{n}$ is equivalent to replacing some $T$ in $\left(T . W_{2}\right)^{n}$ by $\mathscr{C}\left(T ; R^{-1}\right)$ or

replacing $Q$ in $\left(T . W_{2}\right)^{n}$ is equivalent to replacing some $W_{2}$ in $\left(T . W_{2}\right)^{n}$ by $\mathscr{C}\left(W_{2} ; R\right)$. (3.41)

We consider the various forms $Q$ can take.

(a) $Q$ "contains" $T$, that is there is a partition $K . L . T . M . N$ of $\left(T \cdot W_{2}\right)^{n}$ such that $Q=L . T . M,\left(T . W_{2}\right)^{n}=K Q N$ and, for some $\gamma \geqq 1, K . L=\left(T . W_{2}\right)^{n-\gamma}$ and

$$
M . N=W_{2} \cdot\left(T \cdot W_{2}\right)^{\gamma-1} \text {. }
$$


Then we have $R_{1}=L . T . M Y^{-1}$ and $R^{-1}=T\left(W_{1} W_{2} W_{1}\right)^{-1}$. Now clearly replacing $Q$ in $K Q N$ by $\mathscr{C}\left(Q ; R_{1}\right)$ is equivalent to replacing $T$ in $K . L . T . M . N$ by $\mathscr{C}\left(T ; R_{1}\right)$; this in turn is equivalent to replacing $T$ in $K . L . T . M . N$ by $\mathscr{C}\left(T ; R^{-1}\right)$, for by (3.39) and Lemma 2 we have $\mathscr{C}\left(T ; R_{1}\right)=\mathscr{C}\left(T ; R^{-1}\right)$. Hence in this case replacing $Q$ in $K Q N$ is equivalent to replacing $T$ in $K . L . T . M . N$ by $\mathscr{C}\left(T ; R^{-1}\right)$, that is, (3.40) holds.

Since the proofs of the following cases are similar to that of case (a) we shall only give outlines of them.

(b) $Q$ " contains" $W_{2}$, that is, there is a partition $K . L . W_{2} \cdot M \cdot N$ of $\left(T \cdot W_{2}\right)^{n}$ such that $Q=L \cdot W_{2} \cdot M,\left(T \cdot W_{2}\right)^{n}=K Q N$ and, for some $\gamma \geqq 1, K . L=\left(T \cdot W_{2}\right)^{n-\gamma} \cdot T$ and

$$
M . N=\left(T \cdot W_{2}\right)^{\gamma-1} \text {. }
$$

Then (3.41) follows from (3.36), (3.39) and Lemma 2.

(c) $T$ "contains" $Q$. Then (3.40) holds.

(d) $W_{2}$ " contains" $Q$. Then (3.41) holds.

(e) $Q=T_{2}, W_{21}$, where $T=T_{1} T_{2}, W_{2}=W_{21} W_{22}$ and none of $T_{1}, T_{2}, W_{21}, W_{22}$ is the identity. Then, since $l(Q) \geqq\left[\frac{1}{2} l\left(R_{1}\right)\right]$, it follows easily that either $l\left(T_{2}\right) \geqq \frac{1}{6} l\left(R_{1}\right)$ or $l\left(W_{21}\right) \geqq \frac{1}{6} l\left(R_{1}\right)$, so that either (3.40) or (3.41) must hold.

(f) $Q=W_{22}, T_{1}$, where $W_{2}=W_{21} W_{22}, T=T_{1} T_{2}$ and none of $W_{21}, W_{22}, T_{1}, T_{2}$ is the identity. Then, as in (e) above, either (3.40) or (3.41) must hold.

Now $Q$ must take one of the forms considered in (a) to (f) above; hence we have shown that either (3.40) or (3.41) must hold.

$7^{\circ}$. We now suppose that (3.40) holds and obtain a contradiction. We have

$$
\mathscr{C}\left(T ; R^{-1}\right)=W_{1} W_{2} W_{1},
$$

and replacing the $(n-\gamma+1)$ th $T$ in $(T . W)^{n}$ we obtain

$$
l\left\{\left(T . W_{2}\right)^{n}\right\}>l\left\{\left(T \cdot W_{2}\right)^{n-\gamma} \cdot\left(W_{1} W_{2} W_{1}\right) \cdot W_{2} \cdot\left(T \cdot W_{2}\right)^{\gamma-1}\right\} .
$$

Now $\alpha\left(W_{2}, W_{1}\right)=\alpha\left(W_{1}, W_{2}\right)=0$, and, if $W_{1}=I$, then $\alpha\left(W_{2}, W_{2}\right)=0$. Hence (3.42) can be written

$$
l\left\{\left(T . W_{2}\right)^{n}\right\}>l\left\{\left(T \cdot W_{2}\right)^{n-\gamma}\left(W_{1} W_{2} W_{1} W_{2}\right) \cdot\left(T \cdot W_{2}\right)^{\gamma-1}\right\} .
$$

If $n=1,(3.43)$ reduces to

while if $n>1$ we have

$$
l\left(T . W_{2}\right)>l\left\{\left(W_{1} W_{2}\right)^{2}\right\}=l\left(U^{2}\right)
$$

$$
\begin{aligned}
l\left\{\left(T \cdot W_{2}\right)^{n}\right\}=l\left\{\left(T . W_{2}\right)^{n-\gamma} \cdot\left(T \cdot W_{2}\right) \cdot\left(T \cdot W_{2}\right)^{\gamma-1}\right\} \leqq l\left\{\left(T . W_{2}\right)^{n-\gamma}\right\} & +l\left(T \cdot W_{2}\right) \\
& +l\left\{\left(T \cdot W_{2}\right)^{\gamma-1}\right\}-\varepsilon\left(W_{2}, T\right)
\end{aligned}
$$

and

$$
\begin{aligned}
l\left\{\left(T . W_{2}\right)^{n-\gamma}\left(W_{1} W_{2} W_{1} W_{2}\right) \cdot\left(T . W_{2}\right)^{\gamma-1}\right\} \geqq l\left\{\left(T . W_{2}\right)^{n-\gamma}\right\}+l\left\{\left(W_{1} W_{2}\right)^{2}\right\} & +l\left\{\left(T . W_{2}\right)^{\gamma-1}\right\}-\varepsilon\left(W_{2}, T\right),
\end{aligned}
$$


so that (3.44) remains true. Now (3.44) contradicts (3.32) and (3.33) and so we conclude that (3.40) cannot hold.

$8^{\circ}$. We now suppose that (3.41) holds. We have $\mathscr{C}\left(W_{2} ; R\right)=W_{1}^{-1} T W_{1}^{-1}$, and replacing the $(n-\gamma+1)$ th $W_{2}$ in $\left(T . W_{2}\right)^{n}$, we obtain

$$
l\left\{\left(T \cdot W_{2}\right)^{n}\right\}>l\left\{\left(T \cdot W_{2}\right)^{n-\gamma} \cdot T \cdot\left(W_{1}^{-1} T W_{1}^{-1}\right) .\left(T \cdot W_{2}\right)^{\gamma-1}\right\} .
$$

We consider separately the cases $W_{1} \neq I ; W_{1}=I$.

Suppose firstly that $W_{1} \neq I$. Then, since $\alpha\left(T, W_{1}^{-1}\right)=\alpha\left(W_{1}^{-1}, T\right)=0$, we can write (3.45) as

$$
l\left\{\left(T . W_{2}\right)\right\}^{n}>l\left\{\left(T \cdot W_{2}\right)^{n-\nu} \cdot\left(T W_{1}^{-1} T W_{1}^{-1}\right)\left(T \cdot W_{2}\right)^{\gamma-1}\right\} .
$$

If $n=1,(3.46)$ reduces to

while if $n>1$ we have

$$
l\left(T . W_{2}\right)>l\left(T W_{1}^{-1} T W_{1}^{-1}\right)
$$

and

$$
l\left\{\left(T . W_{2}\right)^{n}\right\} \leqq l\left\{\left(T . W_{2}\right)^{n-\gamma}\right\}+l\left(T . W_{2}\right)+l\left\{\left(T . W_{2}\right)^{\gamma-1}\right\}-\varepsilon\left(W_{2}, T\right)
$$

$l\left\{\left(T . W_{2}\right)^{n-\gamma} \cdot\left(T W_{1}^{-1} T W_{1}^{-1}\right)\left(T . W_{2}\right)^{\gamma-1}\right\} \geqq l\left\{\left(T . W_{2}\right)^{n-\gamma}\right\}+l\left\{\left(T W_{1}^{-1} T W_{1}^{-1}\right)+l\left\{\left(T . W_{2}\right)^{\gamma-1}\right\}\right.$

so that (3.47) remains true. From (3.47) we have $-\epsilon\left(W_{2}, T\right)$,

$$
l(T)+l\left(W_{2}\right)>2 l(T)+2 l\left(W_{1}\right),
$$

that is,

$$
l\left(W_{2}\right)>l(T)+2 l\left(W_{1}\right)
$$

Since $\lambda=l(T)+2 l\left(W_{1}\right)+l\left(W_{2}\right)$, it follows that $l\left(W_{2}\right)>\frac{1}{2} \lambda$, which contradicts (3.30). Hence (3.41) cannot hold if $W_{1} \neq I$.

Now suppose that $W_{1}=I$. Then $U=W_{2}, R=W_{2} T^{-1}$ and so

$$
\alpha\left(W_{2}, W_{2}\right)=\alpha\left(W_{2}, T^{-1}\right)=\alpha\left(T^{-1}, W_{2}\right)=0 .
$$

Rewriting (3.32) and (3.33), we have either

$$
l\left(T \cdot \dot{W}_{2}\right)<\left(W_{2}^{2}\right)
$$

or

$$
l\left(T . W_{2}\right)=\left(W_{2}^{2}\right) \quad \text { and } \quad \operatorname{In}(T) \sim \operatorname{Fin}\left(W_{2}\right) .
$$

Suppose firstly that (3.49) holds. Now $l\left(W_{2}\right) \leqq l(T)$ since $W_{2}$ is cyclically $\Omega$-reduced; hence we must have $\varepsilon\left(T, W_{2}\right)=1$. It follows that $\alpha(T, T)=0$, for, if $\alpha(T, T)$ were non-zero, then, since $\varepsilon\left(T, W_{2}\right)=1$, we would have $\alpha\left(T^{-1}, W_{2}\right)>0$, which contradicts (3.48). Hence we can write (3.45) as

$$
l\left\{\left(T . W_{2}\right)^{n}\right\}>l\left\{\left(T . W_{2}\right)^{n-\gamma} \cdot T^{2}\left(T . W_{2}\right)^{y-1}\right\},
$$

from which it follows that

$$
l\left(T . W_{2}\right)>l\left(T^{2}\right)=2 l(T)
$$


Hence $l\left(W_{2}\right)>l(T)$, which contradicts the fact that $W_{2}$ is cyclically $\Omega$-reduced. Thus (3.41) cannot hold if $W_{1}=I$ and (3.49) holds. Now suppose that (3.50) holds. Then again we must have $\alpha(T, T)=0$, since otherwise, as $\varepsilon\left(W_{2}, T\right)=1$, we would have $\alpha\left(W_{2}, T^{-1}\right)>0$, contradicting (3.48). Now we obtain a contradiction from (3.45) as in the previous case. Hence (3.41) cannot hold if $W_{1}=I$ and (3.50) holds. Since either (3.49) or (3.50) must hold we have shown that (3.41) cannot hold if $W_{1}=I$.

Combining our results we have shown that neither (3.40) nor (3.41) can hold. Hence the assumption made in $6^{0}$, that $\left(T . W_{2}\right)^{n}$ contains a subword by which it is $\Omega$-reducible, must be false, that is, $\left(T . W_{2}\right)^{n}$ is $\Omega$-reduced for all positive integers $n$. This completes the proof of the lemma.

The following corollary will be used in a later paper.

COROLlary 2. Let $W$ be cyclically $\Omega$-reduced and $l(W)>1$. Suppose that $W^{2}$ is not cyclically $\Omega$-reduced, so that there exist a cyclic arrangement $U$ of $W$, an element $V$ of $\Pi$ and an element $S$ of $\Omega_{1}$ such that $U^{2}=S V, R=S T^{-1} \in \Omega$ and $l^{0}(T . V)<l\left(U^{2}\right)$. Suppose moreover that $U$ and $V$ are chosen so that $\beta(T, V)=\beta(V, T)=0$. Then, if $|W|$ has infinite order in $\Pi /[\Omega]$, (iii) of the lemma holds with $U=W_{1} W_{2}$ and $V=W_{2}$.

Proof. It is clear that $W$ satisfies (iii) of the lemma, and an examination of the proof of (B), with particular reference to $4^{\circ}$, shows that we must have $U=W_{1} W_{2}$ and $V=W_{2}$.

We now come to the proof of the main theorems.

Proof of Theorem 2. Let $\Pi /[\Omega]$ be an FPS-group such that $\Omega$ contains a proper power $R, R=J^{\lambda}(\lambda>1)$. We note that $l(J) \geqq 2$ and $\alpha(J, J)=0$, since $l(R) \geqq 7$ and $\alpha(R, R)=0$.

We have $J^{\lambda} \approx I$, so that the order $r$ of $|J|$ is less than or equal to $\lambda$. Suppose that $r<\lambda$. Then

$$
J^{\lambda-r}=J^{\lambda}(J)^{-1} \approx I \text { and } \lambda-r>0, \text { so that } r \leqq \lambda-r .
$$

Since $J^{\prime} \approx I$, it must contain a subword $Q$ by which it is $\Omega$-reducible, $J^{\prime}=X Q Y$ say, where $Q T^{-1}=R_{1} \in \Omega$ and $l(Q) \geqq\left[\frac{1}{2} l\left(R_{1}\right)\right]$. Clearly we can find a cyclic arrangement $J_{1}$ of $J$ such that $J_{1}^{r}=Q Y X$. We note that $J_{1}^{\lambda}=Q Y X J_{1}^{\lambda-r} \in \Omega$ since $J^{\lambda} \in \Omega$. Also, since $Q \in \Omega_{1}$, we have, by (2.21) and property (4) of $\Omega$, that

$$
Q T^{-1}=Q Y X J_{1}^{\lambda-r}
$$

Hence $T^{-1}=Y X J_{1}^{\lambda-r}$, and replacing $Q$ in $X Q Y$ we obtain, since $X Q Y=J^{r}$ is reducible by $Q$,

$$
l\left(J^{r}\right)>l\left\{X .\left(J_{1}^{r-\lambda} X^{-1} Y^{-1}\right) . Y\right\} \geqq l^{0}\left\{X .\left(J_{1}^{r-\lambda} X^{-1} Y^{-1}\right) . Y\right\}=l\left(J_{1}^{r-\lambda}\right)=l\left(J^{\lambda-r}\right) .
$$

This is a contradiction, since $r \leqq \lambda-r$. Hence we must have $r=\lambda$, which proves the theorem.

Proof of Theorem 3. Let $\Pi /[\Omega]$ be an FPS-group and let $A$ be an element of $\Pi$ such that $A \neq I$. Then it is easy to see, by an argument similar to that used in the proof of (iii) of Lemma 3, that there exists a cyclically $\Omega$-reduced element $W$ of $\Pi$ such that $|A|$ is conjugate to $|W|$, so that $|A|$ and $|W|$ have the same order. Now, if $l(W)=1$, then by (ii) of Theorem 1 the order of $|W|$ is equal to the order of $W$, while if $l(W)>1$, then by Lemma 5 either $|W|$ has infinite order or (ii) of Lemma 5 holds. (See the note following the statement of Lemma 5.) 


\section{ELEMENTS OF FINITE ORDER IN FREE PRODUCT SIXTH-GROUPS 145}

It follows that, if each constituent group of $\Pi$ is torsion-free and $\Omega$ does not contain a proper power, then $\Pi /[\Omega]$ is torsion-free, while if either $\Pi$ has a constituent group containing elements of finite order or $\Omega$ contains a proper power, then $\Pi /[\Omega]$ has elements of finite order. This proves the theorem.

Proof of Corollary 1. We use the notation of the above proof and suppose that $|A|$ has finite order. It is clear that, if $l(W)=1$, then (i) of the corollary holds, since then we have $|A|=|P|^{-1}|W||P|$ for some $P \in \Pi$, so that $A$ is the image under $\phi$ of $P^{-1} . W . P$. Thus we can suppose that $l(W)>1$. Since $|A|$ has finite order, it follows that (ii) of Lemma 5 must hold, so that there exist $J \in \Pi, R \in \Omega$ and integers $m, n$ such that $W=J^{m}, R=J^{n}$. We may assume that both $m$ and $n$ are positive, since, if $m$ is negative, then $W=\left(J^{-1}\right)^{-m}$ and $R=\left(J^{-1}\right)^{-n}$, while if $n$ is negative, then $W=J^{m}$ and $R^{-1}=J^{-n} \in \Omega$. Let $t$ be the highest common factor of $m$ and $n$. Then $|J|$ has order $n$ by Theorem 2 , and, since $|W|=|J|^{m}$, it follows that $|W|$, and therefore $|A|$, has order $n / t$. This proves the corollary.

\section{REFERENCES}

1. J. L. Britton, Solution of the word problem for certain types of groups I, Proc. Glasgow Math. Assoc. 3 (1956), 45-54.

2. J. L. Britton, Solution of the word problem for certain types of groups II, Proc. Glasgow Math. Assoc. 3 (1956), 68-90.

3. M. Greendlinger, On Dehn's algorithms for the conjugacy and word problems, with applications, Comm. Pure Appl. Math. 13 (1960), 641-677.

4. A Karass, W. Magnus and D. Solitar, Elements of finite order in groups with a single defining relation, Comm. Pure Appl. Math. 13 (1960), 57-66.

5. S. Lipschutz, An extension of Greendlinger's results on the word problem, Proc. Amer. Math. Soc. 15 (1964), 37-43.

UNIVERSITY OF TORONTO

TORONTO, CANADA 